\title{
ON PROJECTIVE MODULES FOR FROBENIUS KERNELS AND FINITE CHEVALLEY GROUPS
}

\author{
CHRISTOPHER M. DRUPIESKI
}

\begin{abstract}
Let $G$ be a simply-connected semisimple algebraic group scheme over an algebraically closed field of characteristic $p>0$. Let $r \geq$ 1 and set $q=p^{r}$. We show that if a rational $G$-module $M$ is projective over the $r$-th Frobenius kernel $G_{r}$ of $G$, then it is also projective when considered as a module for the finite subgroup $G\left(\mathbb{F}_{q}\right)$ of $\mathbb{F}_{q}$-rational points in $G$. This salvages a theorem of Lin and Nakano (Bull. London Math. Soc. 39 (2007) 1019-1028). We also show that the corresponding statement need not hold when the group $G$ is replaced by the unipotent radical $U$ of a Borel subgroup $B$ of $G$.
\end{abstract}

\section{INTRODUCTION}

1.1. Let $G$ be a simply-connected semisimple algebraic group scheme over an algebraically closed field $k$ of characteristic $p>0$. Assume that $G$ is defined and split over $\mathbb{F}_{p}$, and let $F: G \rightarrow G$ be the standard Frobenius morphism defining the $\mathbb{F}_{p}$-structure on $G$. Given an integer $r \geq 1$, let $G_{r}=$ $\operatorname{ker}\left(F^{r}\right)$ be the $r$-th infinitesimal Frobenius kernel of $G$, and let $G\left(\mathbb{F}_{q}\right)=G^{F^{r}}$ be the finite subgroup of $\mathbb{F}_{q}$-rational points in $G$, consisting of the fixed points in $G$ under the $r$-th iterate of $F$. Here $q=p^{r}$. Let $\mathfrak{g}=\operatorname{Lie}(G)$ be the Lie algebra of $G$, and let $u(\mathfrak{g})$ be the restricted enveloping algebra of $\mathfrak{g}$.

In the proceedings of the 1986 Arcata Conference on Representations of Finite Groups, Brian Parshall asked whether a finite-dimensional rational $G$-module that is projective for $G_{1}$ (equivalently, for $\left.u(\mathfrak{g})\right)$ is always projective for $G\left(\mathbb{F}_{p}\right)$ [Par, 5.3]. Lin and Nakano provided an affirmative answer to this question in 1999 by showing that if $M$ is a rational $G$-module, then the complexity $c_{G\left(\mathbb{F}_{p}\right)}(M)$ of $M$ as a $k G\left(\mathbb{F}_{p}\right)$-module is at most one-half the complexity $c_{G_{1}}(M)$ of $M$ as a $G_{1}$-module [LN1, Theorem 3.4]. Since a module is projective if and only if its complexity is zero, this observation answered Parshall's question. The complexities $c_{G\left(\mathbb{F}_{p}\right)}(M)$ and $c_{G_{1}}(M)$ can also be interpreted as the dimensions of the associated cohomological support varieties $\left|G\left(\mathbb{F}_{p}\right)\right|_{M}$ and $\left|G_{1}\right|_{M}$. Thus, the Lin-Nakano approach possesses a certain geometric flavor, and subsequent work by Carlson, Lin, and Nakano [CLN]

Date: November 11, 2018.

2010 Mathematics Subject Classification. Primary 20G10, 20C33; Secondary 20G05, $17 \mathrm{~B} 56$.

The author was supported in part by NSF VIGRE grant DMS-0738586. 
and by Friedlander [Fri1, Fri2] has sought to better understand the relationship between the varieties $\left|G\left(\mathbb{F}_{p}\right)\right|_{M}$ and $\left|G_{1}\right|_{M}$.

In this note we provide an affirmative answer to Parshall's question for all $r \geq 1$. Specifically, given a finite-dimensional rational $G$-module $M$, we show that if $M$ is projective for the $r$-th Frobenius kernel $G_{r}$ of $G$, then $M$ is projective as a $k G\left(\mathbb{F}_{q}\right)$-module where $q=p^{r}$ This generalization was previously claimed by Lin and Nakano in 2007 [LN2, though their argument was incomplete because of an error in the proof of their key proposition; for a more detailed explanation see Section 2.5. The argument we present here is entirely non-geometric in nature, that is, it does not require the use or discussion of support varieties or complexity, but relies instead only on the algebra of distributions on $G$, so is interesting even for the previouslyestablished case when $r=1$. For $r=1$ our argument also eliminates certain assumptions on the prime $p$ that were necessary for the methods of [LN1]. After proving the main theorem, we provide in Section 2.5 an example to show that the corresponding statement need not hold when $G$ is replaced by the unipotent radical $U$ of a Borel subgroup $B$ of $G$. Finally, in Section 3 we discuss some recent results of Friedlander [Fri] that are related to the projectivity of modules over $G\left(\mathbb{F}_{q}\right)$.

1.2. Notation. Let $G, F, G_{r}, G\left(\mathbb{F}_{q}\right)$, and $\mathfrak{g}$ be as defined in Section 1.1. Let $T \subset G$ be a maximal torus defined and split over $\mathbb{F}_{p}$, and let $\Phi$ be the set of roots of $T$ in $G$. Let $B \subset G$ be a Borel subgroup containing $T$ and corresponding to the set $\Phi^{-}$of negative roots in $\Phi$, and let $B^{+} \subset G$ be the opposite Borel subgroup corresponding to the set $\Phi^{+}$of positive roots in $\Phi$. Let $X(T)$ be the integral weight lattice obtained from $T$. Then $X(T)$ is partially ordered by $\lambda \geq \mu$ if and only if $\lambda-\mu$ is a sum of positive roots. Let $U \subset B$ be the unipotent radical of $B$. Set $\mathbb{N}=\{0,1,2, \ldots\}$. Additional notation will be introduced as needed.

\section{The Generalized Parshall Conjecture}

2.1. The algebra of distributions. We begin by recalling certain basic facts concerning the algebra of distributions on $G$; for further details, see [Jan, II.1.12, II.1.19, II.3.3]. Let $\mathfrak{g}_{\mathbb{C}}$ be the complex semisimple Lie algebra of the same Lie type as $G$, with Chevalley basis $\left\{X_{\alpha}, H_{i}: \alpha \in \Phi, i \in[1, n]\right\}$. Let $U\left(\mathfrak{g}_{\mathbb{C}}\right)$ be the universal enveloping algebra of $\mathfrak{g}_{\mathbb{C}}$, and let $U_{\mathbb{Z}}(\mathfrak{g}) \subset U\left(\mathfrak{g}_{\mathbb{C}}\right)$ be the Kostant $\mathbb{Z}$-form of $U\left(\mathfrak{g}_{\mathbb{C}}\right)$. Since $G$ is semisimple and simply-connected, the algebra $\operatorname{Dist}(G)$ of distributions on $G$ with support at the identity, also known as the hyperalgebra of $G$, may be realized as $U_{\mathbb{Z}}(\mathfrak{g}) \otimes_{\mathbb{Z}} k$, the $k$-algebra obtained via scalar extension from $U_{\mathbb{Z}}(\mathfrak{g})$. Thus, Dist $(G)$ admits a $k$-basis consisting of all monomials

$$
\prod_{\alpha \in \Phi^{-}} X_{\alpha, n(\alpha)} \prod_{i=1}^{n} H_{i, m(i)} \prod_{\alpha \in \Phi^{+}} X_{\alpha, n^{\prime}(\alpha)}
$$

\footnotetext{
${ }^{1}$ This statement is referred to in $[\mathrm{LN2}$ as the Generalized Parshall Conjecture.
} 
where $n(\alpha), m(i), n^{\prime}(\alpha) \in \mathbb{N}, X_{\alpha, n}=X_{\alpha}^{n} /(n !), H_{i, m}=\left(\begin{array}{c}H_{i} \\ m\end{array}\right)$, and the products are taken with respect to any fixed ordering of the roots. Similarly, $\operatorname{Dist}(U)$ admits a $k$-basis consisting of all monomials $\prod_{\alpha \in \Phi^{-}} X_{\alpha, n(\alpha)}$ with $n(\alpha) \in \mathbb{N}$. If the integers $n(\alpha)$ are restricted to lie in the range $0 \leq n(\alpha)<$ $p^{r}$, then one obtains a $k$-basis for the algebra $\operatorname{Dist}\left(U_{r}\right)$. Given $\alpha \in \Phi$, the vectors $X_{\alpha, n}$ with $n \in \mathbb{N}$ form a $k$-basis for the algebra $\operatorname{Dist}\left(U_{\alpha}\right)$ of distributions on the one-dimensional root subgroup $U_{\alpha}$, and the vectors $X_{\alpha, n}$ with $0 \leq n<p^{r}$ form a $k$-basis for $\operatorname{Dist}\left(U_{\alpha, r}\right)$, the algebra of distributions on the $r$-th Frobenius kernel $U_{\alpha, r}$ of $U_{\alpha}$.

Each rational $U_{\alpha}$-module $M$ naturally admits the structure of a locally finite $\operatorname{Dist}\left(U_{\alpha}\right)$-module. Moreover, the action of the $X_{\alpha, n}$ on $M$ determines the action of $U_{\alpha}$ on $M$. Indeed, let $x_{\alpha}: \mathbb{G}_{a} \rightarrow U_{\alpha}$ be a fixed isomorphism between the additive group $\mathbb{G}_{a}$ and the root subgroup $U_{\alpha}$. Then for $a \in \mathbb{G}_{a}$, the action of $x_{\alpha}(a) \in U_{\alpha}$ on $m \in M$ is related to the action of the $X_{\alpha, n}$ on $m$ by the equation

$$
x_{\alpha}(a) \cdot m=\sum_{n \geq 0} a^{n}\left(X_{\alpha, n} \cdot m\right) .
$$

2.2. An equality of endomorphism spaces. For each $1 \leq i<q$, define the formal infinite sum

$$
y_{\alpha, i}=\sum_{n \geq 0} X_{\alpha, i+n(q-1)}
$$

and set $y_{\alpha, 0}=1$. Then the $y_{\alpha, i}$ are well-defined operators on any rational $U_{\alpha}$-module.

Lemma 2.1. Let $M$ be a rational $U_{\alpha}$-module. Then the span in $\operatorname{End}_{k}(M)$ of the operators $y_{\alpha, 0}, y_{\alpha, 1}, \ldots, y_{\alpha, q-1}$ is the same as the $k$-span of the operators $\left\{x_{\alpha}(a): a \in \mathbb{F}_{q}\right\}$.

Proof. Since every rational $U_{\alpha}$-module is a sum of finite-dimensional modules, it suffices to assume that $M$ is finite-dimensional. Then there exists an integer $N \geq q$ such that for all $m \in M, X_{\alpha, n} \cdot m=0$ for all $n \geq N$. Then $x_{\alpha}(a)$ acts on $M$ via the finite sum $\overline{x_{\alpha}(a)}:=\sum_{n=0}^{N-1} a^{n} X_{\alpha, n} \in \operatorname{Dist}\left(U_{\alpha}\right)$. Similarly, $y_{\alpha, i}$ acts on $M$ via the finite sum

$$
\overline{y_{\alpha, i}}:=\sum_{\substack{0 \leq m<N \\ m \equiv i \bmod q-1}} X_{\alpha, m},
$$

and the $\overline{y_{\alpha, i}}$ are linearly independent elements of $\operatorname{Dist}\left(U_{\alpha}\right)$.

Let $a \in \mathbb{F}_{q}$. Since $a^{q}=a$, we have $\overline{x_{\alpha}(a)}=\sum_{i=0}^{q-1} a^{i} \overline{y_{\alpha, i}}$, where by convention we set $0^{0}=1$. Writing $\mathbb{F}_{q}=\left\{a_{0}, a_{1}, \ldots, a_{q-1}\right\}$, the matrix for the linear transformation that sends $\overline{y_{\alpha, i}} \mapsto \overline{x_{\alpha}\left(a_{i}\right)}$ is an invertible Vandermonde matrix; cf. [LN2, §3.3]. It follows that $\left\{\overline{y_{\alpha, i}}: 0 \leq i<q\right\}$ and $\left\{\overline{x_{\alpha}(a)}: a \in \mathbb{F}_{q}\right\}$ are each linearly independent sets spanning the same subspace of $\operatorname{Dist}\left(U_{\alpha}\right)$, and consequently that their images span the same subspace of $\operatorname{End}_{k}(M)$. 
2.3. The Generalized Parshall Conjecture for Borel subgroups. We now establish the Generalized Parshall Conjecture for the Borel subgroup $B$ of $G$.

Theorem 2.2. Let $M$ be a finite-dimensional rational B-module. Suppose $M$ is projective as a $B_{r}$-module. Then $M$ is projective as a $k U\left(\mathbb{F}_{q}\right)$-module, and hence also as a $k B\left(\mathbb{F}_{q}\right)$-module.

Proof. Suppose $M$ is projective as a $B_{r}$-module. Then $M$ is a projective $B_{r} T$-module [Jan, II.9.4], and by the explicit description of the projective indecomposable $B_{r} T$-modules [Jan, II.9.5], there exists a $\operatorname{Dist}\left(U_{r}\right)$-basis $\left\{m_{1}, \ldots, m_{s}\right\}$ for $M$ consisting of weight vectors for $T$. Because $U\left(\mathbb{F}_{q}\right)$ is a Sylow $p$-subgroup of $B\left(\mathbb{F}_{q}\right)$, a $k B\left(\mathbb{F}_{q}\right)$-module is projective if and only if it is projective (hence, free) as a $k U\left(\mathbb{F}_{q}\right)$-module. Then to prove the theorem it suffices to show that $\left\{m_{1}, \ldots, m_{s}\right\}$ is also a $k U\left(\mathbb{F}_{q}\right)$-basis for $M$. Since $\operatorname{dim}_{k} \operatorname{Dist}\left(U_{r}\right)=\operatorname{dim}_{k} k U\left(\mathbb{F}_{q}\right)$, to show that $\left\{m_{1}, \ldots, m_{s}\right\}$ is a $k U\left(\mathbb{F}_{q}\right)$ basis for $M$, it suffices to show that the set $\left\{m_{1}, \ldots, m_{s}\right\}$ generates $M$ as a $k U\left(\mathbb{F}_{q}\right)$-module. Using the partial order on $X(T)$, and the operators defined in Section 2.2, we argue by induction on the weight ordering to show that the $k U\left(\mathbb{F}_{q}\right)$-span $M^{\prime}$ of the set $\left\{m_{1}, \ldots, m_{s}\right\}$ contains all weight vectors in $M$, hence is equal to $M$.

To begin, fix an enumeration $\Phi^{-}=\left\{\alpha_{1}, \ldots, \alpha_{N}\right\}$, and let $\lambda \in X(T)$ be a lowest weight of $T$ in $M$. Since $\left\{m_{1}, \ldots, m_{s}\right\}$ is a $\operatorname{Dist}\left(U_{r}\right)$-basis for $M$, it follows that the $\lambda$-weight space $M_{\lambda}$ must be spanned by vectors of the form $X_{\alpha_{1}, q-1} \cdots X_{\alpha_{N}, q-1} \cdot m_{i}$. Since $\lambda$ is a lowest weight vector, we have

$$
X_{\alpha_{1}, q-1} \cdots X_{\alpha_{N}, q-1} \cdot m_{i}=y_{\alpha_{1}, q-1} \cdots y_{\alpha_{N}, q-1} \cdot m_{i} .
$$

Then it follows from Lemma 2.1 that $M_{\lambda} \subseteq M^{\prime}$. Now let $\lambda \in X(T)$ be an arbitrary weight of $T$ in $M$, and set $M_{<\lambda}=\bigoplus_{\mu<\lambda} M_{\mu}$. By induction, $M_{<\lambda} \subseteq M^{\prime}$. On the other hand, $M_{\lambda}$ is spanned by certain vectors of the form $X_{\alpha_{1}, n_{1}} \cdots X_{\alpha_{N}, n_{N}} \cdot m_{i}$ with $0 \leq n_{i}<q$. Given such a vector, the difference

$$
X_{\alpha_{1}, n_{1}} \cdots X_{\alpha_{N}, n_{N}} \cdot m_{i}-y_{\alpha_{1}, n_{1}} \cdots y_{\alpha_{N}, n_{N}} \cdot m_{i}
$$

is an element of $M_{<\lambda}$, so is a vector in $M^{\prime}$. But $y_{\alpha_{1}, n_{1}} \cdots y_{\alpha_{N}, n_{N}} \cdot m_{i} \in M^{\prime}$ by Lemma 2.1, so we conclude that $X_{\alpha_{1}, n_{1}} \cdots X_{\alpha_{N}, n_{N}} . m_{i} \in M^{\prime}$ as well, and hence that $M_{\lambda} \subseteq M^{\prime}$. Since $M$ has only finitely many distinct weight spaces, we conclude that each weight space of $M$ is contained in $M^{\prime}$, and hence that $M=M^{\prime}$. Thus, the set $\left\{m_{1}, \ldots, m_{s}\right\}$ generates $M$ as a $k U\left(\mathbb{F}_{q}\right)$-module.

2.4. Proof of the Generalized Parshall Conjecture. We now recover the main theorem of [LN2], and hence also the results contained in [LN2, $\S \S 3-$ $4]$.

Theorem 2.3. Let $G$ be a connected reductive algebraic group over the finite field $\mathbb{F}_{q}$, and let $M$ be a finite-dimensional rational $G$-module. If $M$ is projective as a $G_{r}$-module, then $M$ is projective as a $k G\left(\mathbb{F}_{q}\right)$-module. 
Proof. By [LN2, Proposition 1.3], it suffices to assume that $G$ is semisimple and simply-connected. Suppose $M$ is projective as a $G_{r}$-module. Since $B_{r}$ is a finite group scheme, the induction functor $\operatorname{ind}_{B_{r}}^{G_{r}}(-)$ is exact [Jan, I.5.13], which implies that $M$ is projective (equivalently, injective) for $B_{r}$ by Jan, I.3.18 and Remark I.4.12]. Now $M$ is projective as a $k U\left(\mathbb{F}_{q}\right)$-module by Theorem 2.2. Since $U\left(\mathbb{F}_{q}\right)$ is a Sylow $p$-subgroup of $G\left(\mathbb{F}_{q}\right)$, this implies that $M$ is projective as a $k G\left(\mathbb{F}_{q}\right)$-module.

2.5. Failure of the Generalized Parshall Conjecture for unipotent subgroups. The following example shows that Theorem 2.3 need not hold if $G$ is replaced by the unipotent radical $U$ of a Borel subgroup $B$ of $G$.

Example 2.4. Suppose $G=S L_{2}$, so that $U \cong \mathbb{G}_{a}$. Then the polynomial $f(t)=t-t^{q}$ defines an algebraic group homomorphism $f: U \rightarrow U$ with $\operatorname{ker}(f)=U\left(\mathbb{F}_{q}\right)$. Now take $M=f^{*}\left(\mathrm{St}_{r}\right)$, that is, the rational $U$-module obtained from the $r$-th Steinberg module $\mathrm{St}_{r}$ by precomposing the $U$-module structure map $U \rightarrow G L\left(\mathrm{St}_{r}\right)$ with $f$. Then $M$ is trivial as a $U\left(\mathbb{F}_{q}\right)$-module. Let $V$ denote the underlying vector space of $\mathrm{St}_{r}$. The $k[U]$-comodule structure maps $\Delta_{\mathrm{St}_{r}}, \Delta_{M}: V \rightarrow V \otimes k\left[\mathbb{G}_{a}\right] \cong V \otimes k[t]$ for $\mathrm{St}_{r}$ and $M$ are related as follows: If $v \in V$ and $\Delta_{\mathrm{St}_{r}}(v)=\sum_{i=0}^{\infty} v_{i} \otimes t^{i}$ with $v_{i} \in V$ and $v_{j}=0$ for all $j \gg 0$, then $\Delta_{M}(v)=\sum_{i=0}^{\infty} v_{i} \otimes f\left(t^{i}\right)=\sum_{i=0}^{\infty} v_{i} \otimes\left(t^{i}-t^{q i}\right)$. It then follows that $M \cong \mathrm{St}_{r}$ as a $\operatorname{Dist}\left(U_{r}\right)$-module, and hence that $M$ is projective as a $U_{r}$-module even though it is trivial for the finite group $U\left(\mathbb{F}_{q}\right)$. Observe that since $f$ is a non-homogenous polynomial, the action of $U$ on $M=f^{*}\left(\mathrm{St}_{r}\right)$ cannot lift to a rational action of the Borel subgroup $B$.

Because of Example 2.4, it follows that Proposition 2.1 and Theorem 2.3 in [LN2 are false for $H=U$, and that [LN2, Corollary 2.4] also does not hold for an arbitrary connected algebraic group defined over $\mathbb{F}_{q}$. The proof of [LN2, Proposition 2.1] fails because, in the notation used there, a homomorphism vanishing on $\operatorname{soc}_{N} Q(L)$ need not be the zero map. Whether or not [LN2, Proposition 2.1] might hold for $H=G$ or $H=B$ remains an open question. Example 2.4 also shows that if $U$ is an arbitrary connected unipotent algebraic group scheme defined over $\mathbb{F}_{q}$, there may exist rational $U$-module structures on $\operatorname{ind}_{1}^{U_{r}}(k)$ that are not projective upon restriction to $U\left(\mathbb{F}_{q}\right)$. It remains an open question whether for such $U$ there always exists some rational $U$-module structure on $\operatorname{ind}_{1}^{U_{r}}(k)$ that is projective upon restriction to $U\left(\mathbb{F}_{q}\right)$; see [LN2, Conjecture 2.4].

\section{Projectivity and Weil Restriction of Restricted Lie ALGEBRAS}

3.1. Restricted Lie algebras arising from filtrations on the group algebra. Let $G$ be as defined in Section 1, and let $M$ be a finite-dimensional rational $G$-module. In their original approach to proving the $r=1$ version of the Parshall Conjecture, Lin and Nakano obtained the inequality $c_{G\left(\mathbb{F}_{p}\right)}(M) \leq \frac{1}{2} c_{G_{1}}(M)$ by first proving that $c_{U\left(\mathbb{F}_{p}\right)}(M) \leq c_{U_{1}}(M)$. To obtain 
the latter inequality, they observed that the group ring $k U\left(\mathbb{F}_{p}\right)$ is filtered by the powers of its augmentation ideal, and that the associated graded algebra $\operatorname{gr} k U\left(\mathbb{F}_{p}\right)$ is isomorphic to the restricted enveloping algebra $u(\mathfrak{u})$ for $\mathfrak{u}=\operatorname{Lie}(U)$. Equivalently, gr $k U\left(\mathbb{F}_{p}\right) \cong \operatorname{Dist}\left(U_{1}\right)$. They then deduced the existence of a spectral sequence $E_{1}^{i, j}=\mathrm{H}^{i+j}\left(U_{1}, M\right)_{(i)} \Rightarrow \mathrm{H}^{i+j}\left(U\left(\mathbb{F}_{p}\right), M\right)$, and from this the inequality $c_{U_{1}}(M) \leq c_{U\left(\mathbb{F}_{p}\right)}(M)$ followed.

In [Fri1, Friedlander applies techniques involving the Weil restriction functor to extend Lin and Nakano's results to the case $r \geq 1$. In this context, the isomorphism gr $k U\left(\mathbb{F}_{p}\right) \cong u(\mathfrak{u})$ is replaced by gr $k U\left(\mathbb{F}_{q}\right) \cong u\left(\mathfrak{u}_{\mathbb{F}_{q}} \otimes_{\mathbb{F}_{p}} k\right)$. Here $\mathfrak{u}_{\mathbb{F}_{q}}$ is the restricted Lie algebra over $\mathbb{F}_{q}$ obtained via scalar extension to $\mathbb{F}_{q}$ from a Chevalley basis for $\mathfrak{u}_{\mathbb{C}}$ (and $\mathfrak{u}_{\mathbb{C}}$ is the obvious Lie subalgebra of $\mathfrak{g}_{\mathbb{C}}$ corresponding to $U$ ). There exists a similar restricted Lie algebra $\mathfrak{u}_{\mathbb{F}}$ with $\mathfrak{u}_{\mathbb{F}_{p}} \otimes_{\mathbb{F}_{p}} \mathbb{F}_{q}=\mathfrak{u}_{\mathbb{F}_{q}}$ and $\mathfrak{u}_{\mathbb{F}_{p}} \otimes_{\mathbb{F}_{p}} k=\mathfrak{u}$. In the isomorphism gr $k U\left(\mathbb{F}_{q}\right) \cong$ $u\left(\mathfrak{u}_{\mathbb{F}_{q}} \otimes_{\mathbb{F}_{p}} k\right)$, the Lie algebra $\mathfrak{u}_{\mathbb{F}_{q}}$ is considered via Weil restriction as a restricted Lie algebra over $\mathbb{F}_{p}$ (by forgetting the additional $\mathbb{F}_{q}$-vector space structure), and then the scalars are extended back to $k$. Replacing $\mathfrak{u}$ by $\mathfrak{g}$, one also has the restricted Lie algebras $\mathfrak{g}_{\mathbb{F}_{p}}, \mathfrak{g}_{\mathbb{F}_{q}}=\mathfrak{g}_{\mathbb{F}_{p}} \otimes_{\mathbb{F}_{p}} \mathbb{F}_{q}$, and $\mathfrak{g}_{\mathbb{F}_{q}} \otimes_{\mathbb{F}_{p}} k$. Since

$$
\mathbb{F}_{q} \otimes_{\mathbb{F}_{p}} \mathbb{F}_{q} \cong \mathbb{F}_{q} \times \cdots \times \mathbb{F}_{q} \quad\left(r \text { times, } q=p^{r}\right),
$$

there exists an isomorphism of restricted Lie algebras $\mathfrak{g}_{\mathbb{F}_{q}} \otimes_{\mathbb{F}_{p}} k \cong \mathfrak{g}^{\oplus r}$.

3.2. Failure of rational modules to be projective. Let $M$ be a rational $G$-module. The action of $G$ on $M$ differentiates to an action of $\mathfrak{g}$, and then restricts to an action of $\mathfrak{g}_{\mathbb{F}_{q}}$ considered as a restricted Lie algebra over $\mathbb{F}_{p}$. This action of $\mathfrak{g}_{\mathbb{F}_{q}}$ on $M$ can be extended over $\mathbb{F}_{p}$ to an action of $\mathfrak{g}_{\mathbb{F}_{q}} \otimes_{\mathbb{F}_{p}} k$ on $M$. Then $\mathfrak{g}_{\mathbb{F}_{q}} \otimes_{\mathbb{F}_{p}} k$ acts on $M$ via the composition of the multiplication map $\mathfrak{g}_{\mathbb{F}_{q}} \otimes_{\mathbb{F}_{p}} k \rightarrow \mathfrak{g}$ with the given action of $\mathfrak{g}$ on $M$. With this convention in hand, Friedlander states the following results:

Theorem 3.1 ((cf. [Fri1, Theorem 4.3])). Let $M$ be a rational G-module. Then

$$
c_{G\left(\mathbb{F}_{q}\right)}(M) \leq \frac{1}{2} c_{u\left(\mathfrak{g}_{\mathbb{F}} \otimes_{\mathbb{F}_{p}} k\right)}(M) .
$$

Corollary 3.2 ((cf. Fri1, Corollary 4.4])). Let $M$ be a rational $G$-module. If $M$ is projective for $u\left(\mathfrak{g}_{\mathbb{F}_{q}} \otimes_{\mathbb{F}_{p}} k\right)$, then $M$ is projective for $k G\left(\mathbb{F}_{q}\right)$.

Identifying $\mathfrak{g}_{\mathbb{F}_{q}} \otimes_{\mathbb{F}_{p}} k$ with $\mathfrak{g}^{\oplus r}$, the induced action of $\mathfrak{g}^{\oplus r}$ on $M$ is obtained by composing the projection $\mathfrak{g}^{\oplus r} \rightarrow \mathfrak{g}$ of $\mathfrak{g}^{\oplus r}$ onto its first factor with the ordinary action of $\mathfrak{g}$ on $M$; this follows from the fact that the multiplication map $k^{\times r} \cong \mathbb{F}_{q} \otimes_{\mathbb{F}_{p}} k \rightarrow k$ is a $k$-algebra homomorphism, and hence identifies with the projection of $k^{\times r}$ onto one of its factors, say, the first. We use this realization for the action of $\mathfrak{g}^{\oplus r}$ on $M$ to show for $r \geq 2$ that a rational $G$-module is never projective over $u\left(\mathfrak{g}_{\mathbb{F}_{q}} \otimes_{\mathbb{F}_{p}} k\right)$, and hence that 
Corollary 3.2 holds vacuously. In particular, this implies that the generalization of the original Lin-Nakano technique to $r>1$ is not an effective method for determining the projectivity of a rational $G$-module over $G\left(\mathbb{F}_{q}\right)$.

Theorem 3.3. Let $M$ be a rational $G$-module, and suppose $r \geq 2$. Then $M$ is not projective for $u\left(\mathfrak{g}_{\mathbb{F}_{q}} \otimes_{\mathbb{F}_{p}} k\right)$.

Proof. Let $M$ be a rational $G$-module, and identify $\mathfrak{g}_{\mathbb{F}_{q}} \otimes_{\mathbb{F}_{p}} k$ with $\mathfrak{g}^{\oplus r}$. Suppose $r \geq 2$. Denote the $p$-th power map on $\mathfrak{g}$, that is, the map defining the structure of a $p$-restricted Lie algebra on $\mathfrak{g}$, by $x \mapsto x^{[p]}$. Choose $0 \neq$ $x \in \mathfrak{g}$ with $x^{[p]}=0$, and set $z=(0, x, 0, \ldots, 0) \in \mathfrak{g}^{\oplus r}$. Then $z^{[p]}=0$. Since $\mathfrak{g}^{\oplus r}$ acts on $M$ via the first-factor projection map $\mathfrak{g}^{\oplus r} \rightarrow \mathfrak{g}$ composed with the given action of $\mathfrak{g}$ on $M$, one has $z \cdot M=0$. Let $u(z) \cong k[t] /\left(t^{p}\right)$ be the cyclic subalgebra of $u\left(\mathfrak{g}^{\oplus r}\right)$ generated by $z$. Then $M$ is trivial as a $u(z)$-module, hence not projective over $u(z)$, since every projective $u(z)$ module is free. This implies by [FP1, Corollary 1.4] that the support variety $\left|\mathfrak{g}^{\oplus r}\right|_{M}$ is nonzero, hence by [FP2, Proposition 1.5] that $M$ is not injective (equivalently, projective) for $u\left(\mathfrak{g}^{\oplus r}\right)=u\left(\mathfrak{g}_{\mathbb{F}_{q}} \otimes_{\mathbb{F}_{p}} k\right)$.

\section{REFERENCES}

[CLN] J. F. Carlson, Z. Lin, and D. K. Nakano, Support varieties for modules over Chevalley groups and classical Lie algebras, Trans. Amer. Math. Soc. 360 (2008), no. 4, 1879-1906.

[Fri1] E. M. Friedlander, Weil restriction and support varieties, J. Reine Angew. Math. 648 (2010), 183-200.

[Fri2] E. M. Friedlander, Restrictions to $G\left(\mathbb{F}_{p}\right)$ and $G_{(r)}$ of rational $G$-modules, Compositio Math. 147 (2011), 1955-1978.

[FP1] E. M. Friedlander and B. J. Parshall, Support varieties for restricted Lie algebras, Invent. Math. 86 (1986), no. 3, 553-562.

[FP2] Geometry of p-unipotent Lie algebras, J. Algebra 109 (1987), no. 1, 25-45.

[Jan] J. C. Jantzen, Representations of algebraic groups, second ed., Mathematical Surveys and Monographs, vol. 107, American Mathematical Society, Providence, RI, 2003.

[LN1] Z. Lin and D. K. Nakano, Complexity for modules over finite Chevalley groups and classical Lie algebras, Invent. Math. 138 (1999), no. 1, 85-101.

[LN2] - Projective modules for Frobenius kernels and finite Chevalley groups, Bull. Lond. Math. Soc. 39 (2007), no. 6, 1019-1028.

[Par] B. J. Parshall, Cohomology of algebraic groups, The Arcata Conference on Representations of Finite Groups (Arcata, Calif., 1986), Proc. Sympos. Pure Math., vol. 47, Amer. Math. Soc., Providence, RI, 1987, pp. 233-248.

Department of Mathematics, DePaul University, Chicago, IL 60614

E-mail address: cdrupies@depaul.edu 